\title{
Following on from the Life Esidimeni incident - access to care for people living with severe mental disability, according to national policy
}

\author{
B Janse van Rensburg, MB ChB, FCPsych(SA), MMed, PhD \\ Department of Psychiatry, Faculty of Health Sciences, University of the Witwatersrand, Johannesburg; President, South African Society of Psychiatrists \\ (2016-2018)
}

Corresponding author: B Janse van Rensburg (albert.jansevanrensburg@wits.ac.za)

\begin{abstract}
Following the release in February 2017 of the health ombudsman's report on the deaths of psychiatric patients in Gauteng Province, South Africa (SA) (the Life Esidimeni incident), the South African Society of Psychiatrists (SASOP) has called for a complete overhaul of the mentalhealthcare system in terms of the principles of existing mental-health policy, in particular the National Mental Health Policy Framework and Strategic Plan 2013 - 2020 (NMHPF and SP). Overhauling the mental-healthcare system according to the NMHPF and SP will include the identification and costing of the required facility and staffing interventions that must be put into place to ensure capacity and integration on the different service levels, namely: residential and day-care community-based facilities and programmes, providing a range of care, treatment and rehabilitation services with a fulltime multidisciplinary team available; primary mental-healthcare services at district level; a specialist community-psychiatry service; acute psychiatric units in secondary, tertiary and central general hospitals; and specialised psychiatric beds.
\end{abstract}

Following on from the Life Esidimeni tragedy, where more than 100 psychiatric patients died because of a poorly planned de-institutionalisation project implemented by the Gauteng Department of Health (GDoH), and in view of ongoing pressures on the mental healthcare delivery system in Gauteng Province and elsewhere, the South African Society of Psychiatrists (SASOP) called in February 2017, and again in June 2017, for a complete overhaul of the mentalhealthcare system. ${ }^{[1,2]}$ It was noted in these SASOP statements that such an overhaul should be considered in terms of the principles of existing mental-health policy, in particular the National Mental Health Policy Framework and Strategic Plan 2013 - 2020 (NMHPF and SP). ${ }^{[3]}$

\section{Discussion}

This involves the integration of services on the different levels (Fig. 1), ${ }^{[3]}$ namely: (i) residential and day-care community-based facilities and programmes, providing a range of care, treatment and rehabilitation services with a full-time multidisciplinary team; (ii) primary mentalhealthcare services in districts; (iii) specialist community psychiatry service; (iv) acute psychiatric inpatient units in secondary, tertiary and central general hospitals; and $(v)$ specialised psychiatric beds.

\section{Residential and day-care community-based facilities and programmes}

According to the NMHPF and SP (Table 1 ) ${ }_{1}^{[3]}$ provincial departments of health will license and regulate the provision of community-based mental-health services by non-governmental organisations (NGOs) and for-profit organisations, such as community residential care, daycare services, and halfway houses.

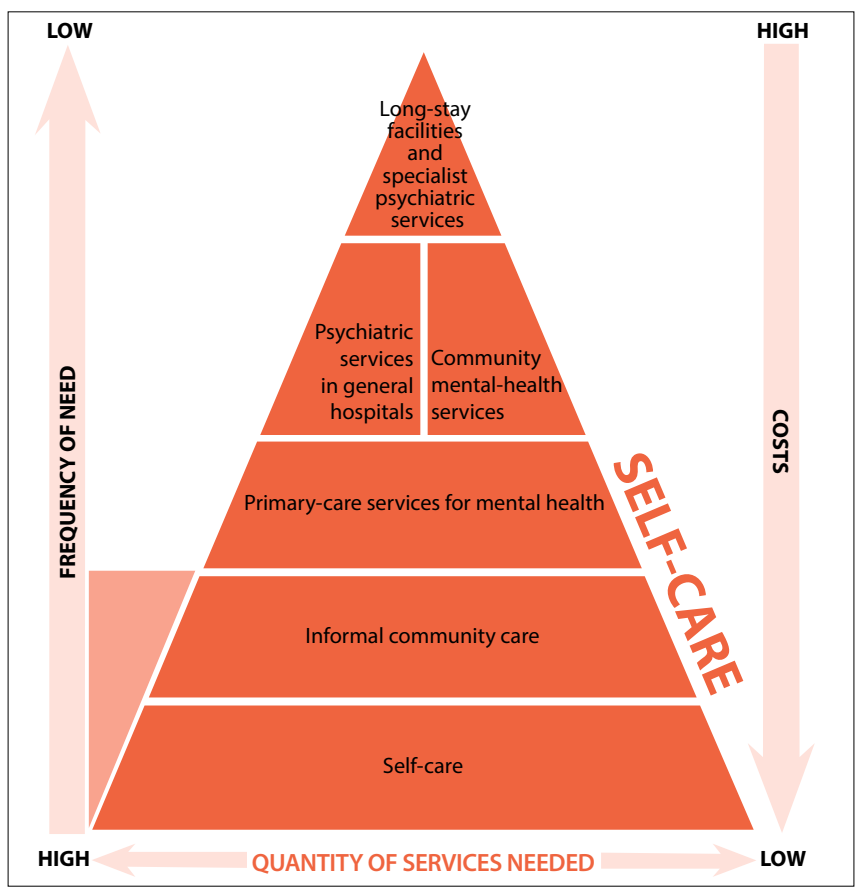

Fig. 1. Organisation of services in the National Mental Health Policy Framework and Strategic Plan $2013-2020 .^{[3]}$

\section{Primary mental-healthcare services in districts}

This represents the first level of contact for individuals seeking healthcare in terms of the NMHPF and SP (p8). ${ }^{[3]}$ Primary clinical psychiatric care may include the management of mild depression 
Table 1. Residential and day-care community-based facilities and programmes: Definitions and terms according to NMHPF and $\mathrm{SP}^{[3]}$

\begin{tabular}{ll}
\hline $\begin{array}{l}\text { Primary healthcare (PHC) and primary-level services } \\
\text { (page 8) }\end{array}$ & $\begin{array}{l}\text { Community mental-health services (page 23) } \\
\text { These will be scaled up, to match recommended national norms, and will }\end{array}$ \\
$\begin{array}{l}\text { PHC: Essential healthcare is to be made accessible at a cost } \\
\text { the country and community can afford, with methods that are }\end{array}$ & $\begin{array}{l}\text { include three core components: (i) community residential care (including } \\
\text { assisted living and group homes); (ii) day-care services; and (iii) outpatient }\end{array}$ \\
practical, scientifically sound and socially acceptable (Alma Ata & $\begin{array}{l}\text { services (including general health outpatient services in PHC and specialist } \\
\text { Declaration, 1978). This approach is organised to reduce exclusion } \\
\text { mental-health support). These community mental-health services will } \\
\text { and social disparities in health, is people-centred, intersectoral and } \\
\text { be developed before further downscaling of psychiatric hospitals can }\end{array}$ \\
$\begin{array}{l}\text { collaborative, and promotes the participation of all stakeholders. } \\
\text { proceed. In accordance with MHCA, NGOs and voluntary and consumer }\end{array}$ \\
$\begin{array}{l}\text { primary-level services: The first level of contact for individuals } \\
\text { seeking healthcare. }\end{array}$ & $\begin{array}{l}\text { organisations will be eligible to provide and be funded for community } \\
\text { programmes/facilities. This includes capacity development for users }\end{array}$ \\
& $\begin{array}{l}\text { (service users and their families) to provide appropriate self-help and peer- } \\
\text { led services, for example as community health workers. }\end{array}$
\end{tabular}

Table 2. Primary mental-healthcare services in districts: Definitions and terms according to NMHPF and SP ${ }^{[3]}$

\begin{abstract}
The district mental-health system will be strengthened in the following areas (page 23):
(i) Specified mental-health interventions will be included in the core package of district health services, embracing a task-shifting approach whereby trained non-specialist workers deliver evidence-based psychosocial interventions. These should include medication monitoring and psychosocial rehabilitation within a recovery framework for severe mental illness; detection and a stepped approach to management and referral of depression and anxiety disorders in PHC clinics; detection and management of child and adolescent mental disorders in PHC clinics and at community level (e.g. schools), and referral where appropriate; routine screening for mental illness during pregnancy; and a stepped approach to management and referral.
\end{abstract}

(ii) Mental-health training programmes for general health staff will be conducted at PHC level and district and regional hospitals.

(iii) Supervision systems will be put in place for mental-health staff at PHC level.

(iv) Specialist mental-health teams will be established to support non-specialist PHC staff and community-based workers.

(v) Clinical protocols will be available for assessment and interventions at PHC level, through integrated management guidelines, which will include mental health.

(vi) Community-based rehabilitation programmes will be established in all districts, using a task-shifting approach.

(viii) Mechanisms will be developed for intersectoral collaboration for mental health, led by the health sector and engaging a range of other sectors. (ix) Inpatient units will be built in district and regional hospitals.

(x) Voluntary mental-healthcare users who require admission will be admitted in terms of general health legislation.

(xi) Assisted and involuntary mental-healthcare users will be admitted in terms of the provisions and procedures described in the MHCA as emergency admissions, or for 72-hour assessment in facilities that are listed for this purpose. Further care, treatment and rehabilitation of such users will be provided at health establishments designated for this purpose in terms of the MHCA.

Roles and responsibilities of district health services (page 31)

(i) Providing mental-health promotion and prevention interventions, in keeping with national and provincial priorities; (ii) inclusion of mental health in the core package of district health treatment and rehabilitation services (e.g. routine screening for mental illness during pregnancy, and provision of counselling and referral where appropriate; medication monitoring and psychosocial rehabilitation within a recovery framework for severe mental illness; detection of mental illness and management of common mental disorders such as depression and anxiety disorders in PHC clinics, and referral where appropriate; and detection and management of child and adolescent mental disorders in PHC clinics, and referral where appropriate; (iii) providing 24-hour emergency care and 72-hour observation services in designated district and regional hospital inpatient settings, as set out in the MHCA; (iv) conducting mental-health training programmes for all general health staff for basic screening, detection and treatment, as well as referral of complex cases; $(v)$ establishing and maintaining mental-health supervision systems for health staff at PHC level; (vi) establishing and maintaining specialist mental-health teams to support PHC staff; (vii) establishing and maintaining referral and back-referral pathways for mental health; (viii) implementing clinical protocols for assessment and interventions at PHC level; (ix) establishing and maintaining community-based rehabilitation programmes, through trained community health workers; $(x)$ developing intersectoral collaboration between a range of sectors involved in mental health, through the establishment of district multisectoral forums for mental health; (xi) undertaking mentalhealth education programmes in communities; (xii) improving the capacity of district health management teams for planning, implementing, supervising, monitoring and evaluation of mental-health programmes at district and community levels; and (xiii) providing of psychotropic medication to all appropriate levels of the district health system, as determined by the essential drugs list.

$\mathrm{PHC}=$ primary healthcare

and anxiety, the maintenance of stabilised psychotic conditions and of substance use and abuse, including intoxication and followup of psychosis/mood problems related to substances, as well as adjustment disorders. Primary care, level-I district hospitals and family-medicine specialists in the district are described accordingly in the NMHPF and SP (Table 2).

\section{Specialist community-psychiatry services in the district}

District specialist mental-health teams consist of a psychiatrist, psychologist, occupational therapist, specialist professional nurse and social worker. For terms of reference, refer to NMHPF and SP (Table 3). 
Table 3. Specialist community-psychiatry services in the district: Definitions and terms according to NMHPF and SP ${ }^{[3]}$

Specialist community psychiatry service in the district, terms of reference (Appendix 1, page 41)

(i) Adopt a public-health approach to the mental health of the district, conducting a situation analysis of mental-health needs and service resources in the district population, and developing an action plan for promotion, prevention, treatment and recovery. (ii) Establish routine ongoing training and supervision for PHC staff through the district specialist mental-health team. Establish routine referral pathways from primary care to specialist services in each district.

(iii) Introduce routine indicated assessment and management of common mental disorders (depression, anxiety and alcoholuse disorders) in priority programmes at PHC level: TB; HIV/AIDS; antenatal mothers; postnatal care; family planning; and chronic diseases.

(iv) Embed suicide prevention in treatment at PHC level, through identification of risk factors for suicide in all health-service provision. (v) Strengthen school systems for mental-health promotion, prevention of mental illness, detection and management of child and adolescent mental disorders in schools, and referral where appropriate, in line with the School Health Policy.

(vi) Establish posts for psychologists in community settings, and look for opportunities for psychologists in psychiatric hospital settings to move to community settings.

(vii) Provide clinical and consultation liaison services within the district.

(viii) Encourage the implementation of the Traditional Health Practitioners Act No. 22 of 2007, by facilitating links between mental-health services and traditional healers and faith healers at local district levels, including appropriate referral pathways in both directions.

(ix) Deploy intern psychologists and registered counsellors to provide training, supervision and support for the counselling roles of community health workers.

(x) Build capacity for users (service users and their families) to provide appropriate self-help and peer-led services, such as support groups, facilitated by NGOs.

\section{Acute psychiatric units in secondary, tertiary and central general hospitals}

Psychiatric services/units in general hospitals render specialist levels of services back-to-back with community psychiatric health services (Table 4). According to the Mental Health Care Act No. 17 of 2002 (MHCA), 72-hour assessment services are also rendered in general hospitals, including district hospitals providing primary care service level.

- Secondary hospitals. While secondary clinical psychiatric care may include routine maintenance and review of psychiatric conditions, such as mood, psychotic, anxiety, cognitive and other disorders, a regional hospital provides identified specialist services, including psychiatry on a 24-hour basis, and a defined regional drainage population within provincial boundaries. They have $400-800$ beds. ${ }^{[4]}$ It also receives outreach from tertiary hospitals.

- Tertiary and central hospitals. Tertiary clinical psychiatric care includes: treatment-resistant conditions; the failure of first and second line of treatment; medical comorbidity requiring liaison with other specialists or subspecialists, e.g. cardiovascular instability, thyroid abnormality, rheumatology, neurology, pain
Table 4. Acute psychiatric units in secondary, tertiary and central general hospitals: Definitions and terms according to NMHPF and SP[3]

Secondary and tertiary care (Glossary, page 8)

Secondary care: Specialist care that is typically rendered in a hospital setting following a referral from a primary or community health facility.

Tertiary care: Specialist care that is rendered at central hospitals.

Psychiatric services in general hospitals (page 24)

(i) Inpatient units will be provided in general hospitals to improve access for voluntary admission, assisted care, emergency mentalhealth services, 72-hour assessment of involuntary mentalhealthcare users, further care, treatment and rehabilitation. (ii) Voluntary mental healthcare users who require admission will be admitted in terms of general health legislation.

(iii) The psychiatric wards that are attached to general hospitals must be designated in terms of the MHCA where they meet the criteria. (iv) The general hospitals that provide 72-hour assessment for involuntary mental healthcare must be listed as prescribed in the general regulations of the MHCA.

(v) Information regarding health establishments that provide 72-hour assessment for involuntary mental healthcare must be compiled and provided to relevant stakeholders to facilitate referral and access to services.

MHCA $=$ Mental Health Care Act No. 17 of 2002

and obstetrics and gynaecology; psychiatric subspecialty areas in a hospital setting; and psychotherapy in a specialist hospital setting. A tertiary hospital provides specialist and subspecialist services, and has $400-800$ beds. $^{[4]}$ A central hospital provides tertiary and central referral services, and highly specialised and super-specialised national referral units. It has 1200 or more beds, while both tertiary and central hospitals provide training attached to a medical school, and also conduct research. ${ }^{[4]}$

\section{Specialised psychiatric beds}

The definition of a specialised hospital is a facility that providespsychiatry, tuberculosis, infectious diseases or rehabilitation services, and has 600 or more beds..$^{[4]}$ The care provided in specialised psychiatric hospitals and rehabilitation centres is described in the NMHPF and SP (Table 5).

\section{Conclusion}

The NMHPF and SP, with its eight objectives, resulted from the allimportant national summit on mental health, held in April 2012, and the associated Ekurhuleni Declaration on Mental Health (Appendix 3 of the NMHPF and SP, pp $48-51) .{ }^{[3]}$ SASOP commended this policy document at the time, and has commented in detail on its different components. ${ }^{[5]}$ While the $\mathrm{GDoH}$, with the decision to terminate its contract with Life Esidimeni, has allowed all the principles of these commitments to be ignored, there are renewed calls to provinces to ensure the actual implementation of this policy in Gauteng and elsewhere and to urgently translate it into practical action plans, with enabling budgets to fund the required facilities and staffing.

In terms of the strategic action to take, the way forward would include the tasks listed below.

(i) Organisation and management: Management forums designated to mental health and psychiatry must be established for tertiary and central-hospital drainage areas, and between levels of service. 
Table 5. Specialised psychiatric beds: Definitions and terms according NMHPF and SP ${ }^{[3]}$

\section{Specialised (designated) psychiatric hospitals and}

rehabilitation centres (pages $\mathbf{2 4}$ and 32)

(i) Provide further care, treatment and rehabilitation of mental healthcare users in specialised psychiatric hospitals.

(ii) Provide inpatient and limited outpatient mental-health services. (iii) Function as centres of excellence that provide ongoing routine training, supervision and support to secondary and PHC services. (iv) Provide subspecialist services, such as forensic psychiatry and child and adolescent services.

(v) Enable forensic facilities to fulfil their role as set out in the Criminal Procedure Act No. 51 of 1977 as amended, with regards to forensic psychiatric observations. Sections 41 and 49 of the MHCA provide for the designation of health establishments and procedures with regards to state patients and mentally ill prisoners.

$\mathrm{PHC}=$ primary healthcare; $\mathrm{MHCA}=$ Mental Health Care Act No. 17 of 2002

(ii) Facilities: Facilities on the different levels must be brought up to an acceptable standard, including identified projects at acute specialist units as well as others, such as 72-hour assessment facilities at district hospitals.

(iii) Human resources: Core posts must be identified to allow for the appointment of adequate staff on the different levels. (iv) Budget review and strategic planning: The proportion of the health budget that is available for mental healthcare must be identified, with a view to following 1- and 5-year strategic planning cycles.

(v) Implementation task teams and workshops: Provincial task teams must be appointed to operationalise and implement national mental-healthcare policy in terms of the NMHPF and SP.

1. South African Society of Psychiatrists (SASOP) Media Statement. Call for Complete Overhaul of Mental Health Care System. https://www.sasop.co.za/Content/ Images/StatementHealthOmbud_LE4Feb2017.pdf (accessed 8 February 2017).

2. South African Society of Psychiatrists (SASOP) Media Statement. Call for Access to Care for People Living with Severe Mental Disability. https://www.sasop.co.za/ Content/images/SASOPSTATEMENTPressRelease.pdf (accessed 28 June 2017).

3. National Department of Health. National Mental Health Policy Framework and Strategic Plan 2013 - 2020. Pretoria: NDoH, 2013. https://www.health-e.org.za/ wp-content/uploads/2014/10/National-Mental-Health-Policy-Framework-andStrategic-Plan-2013-2020.pdf (accessed 3 June 2017).

4. Regulations relating to categories of hospitals. Government Gazette, 12 August 2011, R655.

5. Janse van Rensburg ABR. Contributions from the South African Society of Psychiatrists (SASOP) to the National Mental Health Action Plan. S Afr J Psych 2013;19(4):205-212. https://doi.org10.7196/SAJP.501

Accepted 17 October 2017 